Ophthalmologica 1988;197:219-220

\title{
Subject Index
}

Accommodation 65

Age-opacity relationship 14

AIDS 113,169

Alcohol 159

Alkali burn 179

Alpha human atrial natriuretic peptides 104

ALT 166

Amblyopia 127

Angioid streaks 36

Aniseiconia 7

Autopsy eye 208

B-Blocking agent 90

Blood osmolality 60

Body weight 60

Bovine-factor-VПI-derived polypeptide 51

Campimetry 151

Cavernous hemangioma, retina 127

Cerclage 47

Choroid ischemia 113

Choroidal vessels 34

Ciliary processes 104

Coat's disease 185

Colors 159

Concomitance 57

Cone dystrophy 57

Conjunctiva 176

Contact lenses 7,166

Contrast sensitivity function 159

Cornea 179

Corneotrabecular endothelium 169

Corrected loss variance 136,144

Cotton-wool-type spots 113

Crystalline lenses 130

Cutting device 110

Cystic fibrosis 202

Cytomegalovirus 169

Diplopia 75

Endophthalmitis 19 Epidemiologic study 26 Epidemiology 69

Epidermal growth factor 179 Epithelium 179 Exotropia 127 
Exudative vasoculopathy 185 Eye movement 85

Fibronectin 179 FITC-polypeptide fraction 51 Fluorescein angiography 51 Foam cell 120

Fundus changes 26

Glaucoma 69, 90, 136, 144, 151, 166 Granular dystrophy 57 Grave's ophthalmopathy 75

Hamartoma 42 Hemodialysis 60

Inclusion cysts 176 Industrial injuries 97 In the bag fixation 208 Intraocular foreign body 97

pressure $60,69,104$

tumor 120

Kaposi's sarcoma 113

Laser 166

- $\quad$ treatment 65

Lens back light scatter 14

- density 14

Lens Opacity Meter 70114 Levobunolol 90

Macrophage 120 Macula 34

Macular degeneration 36 Magnetic resonance imaging 193 Malignant melanoma 176 Mean

defect 144 Memory-guided saccades 85 Metabolic disease 1 Mydriasis 65 Myopia 65

220

Subject Index

Necrotizing retinitis 113

Nevus 176

NOSPECS classification 75

Oblique effect 159 Ocular lateropulsion 85

- pathology 110

Optic nerve head drusen 36

- $\quad$-, inflammation, demyelination, atrophy 193Out of the bag fixation 208

Paget's disease, bone 36

Panretinal photocoagulation 65

Panuveitis 169

Pars plana vitrectomy 19

Perforating ocular wounds 97

Periphlebitis 113

Polydioxanone 47

Posterior chamber intraocular lens 208

Prednisone 75

Prevention 97

Proptosis 75

Pseudophakia 19

Pseudoxanthoma elasticum 36

Radioautography 104 Regeneration 179 Retina 42 Retinal detachment 120

pigment epithelium 42

tears 65

telangiectasis 185

vein occlusion 69

Retinitis pigmentosa 130,185 Retrobulbar irradiation 75 
- $\quad$ neuritis 193Richner-Hanhart syndrome 1

Scatter 159

Sickle cell anemia 36

- $\quad$ - disease 26Stereoscopic vision 7Subretinal fluid 120

Taurine clearance 130 Tear film 202

flow 60

mucus ferning 202 Therapeutic outcome 75 Transmission electron microscopy 20 Tyrosinemia type II 1

Unilateral aphakia 7

Video screen 151 Visual acuity 75

field indices 136,144

fields 136, 144 Vitreoretinal interrelationships 110 Vitreous hemorrhage 127 Vortex veins 34

W. Wallenberg's syndrome 85

YLT 166 